\section{Aspectos éticos de los ensayos clínicos en los países en desarrollo}

Los aspectos éticos de los ensayos clínicos patrocinados o realizados por instituciones de países desarrollados y llevados a cabo en países en desarrollo son un tema controvertido. La Comisión Asesora Nacional de Bioética de los Estados Unidos de América ha abordado recientemente estos problemas y en este artículo, los autores, presidente y director ejecutivo de dicha comisión, analizan algunos de esos aspectos, en particular los relacionados con los ensayos clínicos de fase 3.

Los ensayos clínicos no deben explotar a los individuos que acepten participar en ellos. Estados Unidos (EE. UU.) es uno de los países que han elaborado normas éticas relacionadas con la realización de ensayos clínicos y que disponen de procedimientos para llevar a la práctica dichas normas, basadas en los principios de justicia y autonomía del individuo. Aunque no está exento de deficiencias, este sistema ha funcionado razonablemente bien con las investigaciones clínicas realizadas en los EE. UU., pero con respecto a los ensayos clínicos patrocinados por este país y realizados en países en desarrollo se plantean dos cuestiones: si se les deben aplicar las mismas normas y procedimientos éticos que a los realizados en los EE. UU., a pesar de que el contexto puede ser diferente, y si plantean problemas éticos especiales que hay que solucionar.

Los autores creen que los estudios clínicos realizados en el extranjero deben cumplir todas las normas éticas requeridas para los realizados en los EE. UU., incluidas la revisión y aprobación previa por comités de ética, la minimización del riesgo para los participantes, la exigencia de una relación favorable entre los riesgos y los beneficios y la obtención del consentimiento informado de todos los participantes. Además, debería haber normas adicionales para lograr que los participantes reciban una atención médica adecuada y que las lesiones directamente relacionadas con la participación en el estudio sean recompensadas, aunque esto no sea necesario en los estudios realizados en los EE. UU. No obstante, los procedimientos adoptados para garantizar el cumplimiento de todas estas normas pueden ser diferentes en los países en desarrollo, ajustándose a la cultura, costumbres y condiciones locales.

Otra importante salvaguardia consiste en lograr que los estudios respondan a las necesidades sanitarias de los países donde se realizan, que la intervención investigada sea costeable para el país y que la infraestructura sanitaria nacional soporte su uso y distribución, con el fin de conseguir que los participantes puedan disfrutar de los potenciales beneficios de la intervención.

Por consiguiente, los investigadores, promotores y comités de ética de los países desarrollados deben tomar todas las precauciones para que las justificaciones para realizar un ensayo clínico en un país en desarrollo estén bien fundamentadas. Esto es especialmente importante cuando se trata de países cuya población es especialmente vulnerable a la explotación, debido a la pobreza, a la enfermedad o a la falta de conocimientos para comprender los temas científicos relacionados con el problema a estudiar y el papel del ensayo clínico en la búsqueda de soluciones. La realización de un ensayo clínico en un país en desarrollo porque resulte más cómodo, eficiente o menos problemático no será nunca justificación suficiente.

Los procedimientos para obtener el consentimiento voluntario e informado en los países en desarrollo pueden tener que ser adaptados a las costumbres y cultura locales. En los países desarrollados, el procedimiento legal se centra más en el documento de consentimiento en sí mismo que en el proceso de obtención del consentimiento, que debe ser dado por escrito. En los países en desarrollo, esto puede ser imposible debido al analfabetismo y una alternativa éticamente sólida consiste en la obtención del consentimiento oral ante testigos. De cualquier forma, sea en un país desarrollado o en desarrollo, la obtención de un papel firmado no es garantía de que el paricipante haya comprendido los objetivos de la investigación.

Uno de los temas más controvertidos relacionados con la realización de ensayos clínicos en los países en desarrollo consiste en determinar si el grupo de control debe recibir la misma intervención que se le proporcionaría en un país desarrollado. A juicio de los autores, la intervención experimental debe compararse con un tratamiento de eficacia establecida, independientemente de que esté disponible o no en el país donde se realice el estudio. No obstante, pueden permitirse excepciones, pero en estos casos los investigadores y los promotores deben asumir la responsabilidad de justificar desde el punto de vista ético la necesidad de esas excepciones. 
La revisión por comités de ética del país promotor y del país donde se vaya a realizar la investigación no garantiza que el ensayo clínico se realice de forma ética, pero contribuye a que se tomen en consideración tanto los aspectos éticos del ensayo clínico como el contexto local. En este sentido, hay que redoblar los esfuerzos para conseguir que los comités de ética locales tengan los conocimientos necesarios para afrontar sus responsabilidades.

Aunque se ha señalado que no es ético realizar ensayos clínicos en países que no disponen de regímenes políticos democráticos y que la Administración de Alimentos y Medicamentos (FDA) de los EE. UU. no debería permitir la exportación para ensayos clínicos de fármacos cuyo uso con este fin no haya sido aprobado en EE. UU., los autores creen que estas posturas son demasiado rígidas e impedirían la realización de investigaciones éticamente sólidas.

Otra importante obligación ética consiste en poner a disposición de los participantes en un ensayo clínico las nuevas intervenciones que hayan tenido éxito, y ya se han tomado medidas para que esto sea siempre así. Aunque la obligación ética de proporcionar la intervención al resto de la comunidad que pueda beneficiarse de ella no es tan perentoria, las medidas destinadas a conseguir este objetivo también ayudarían a reducir el riesgo de explotación.

En resumen, los autores creen que los ensayos clínicos realizados en países en desarrollo deberían atenerse a las mismas normas que se utilizarían en los países desarrollados promotores, aunque se pueden permitir algunas excepciones y adaptaciones a las necesidades locales. (Shapiro HT, Meslin EM. Ethical issues in the design and conduct of clinical trials in developing countries. N Engl J Med 2001;345:139-142.)

\section{La lactancia materna y la mortalidad infantil en América Latina}

La baja prevalencia y la baja duración de la lactancia materna se asocian con un aumento del riesgo de morbilidad y mortalidad en lactantes, sobre todo por enfermedades diarreicas e infecciones respiratorias agudas. Sin embargo, los estudios publicados acerca de este tema tienen varios problemas metodológicos. Por ejemplo, la lactancia materna ha sido analizada generalmente como una variable dicotómica y a veces no ha sido definida claramente. Además, aunque el riesgo de mortalidad asociado con la lactancia no materna depende en gran medida de la edad, la mayoría de los estudios no han diferenciado a los lactantes en alto y bajo riesgo, lo cual dificulta la cuantificación de los beneficios de la lactancia exclusivamente materna y la comparación de los beneficios obtenidos dependiendo de la edad del lactante. Los autores de este estudio han investigado el efecto que sobre la mortalidad infantil tienen la lactancia exclusivamente materna durante los 3 primeros meses de vida y la lactancia parcialmente materna desde entonces hasta el año en América Latina y el Caribe.

Los datos sobre la lactancia materna se obtuvieron de encuestas recientes con representatividad nacional realizadas en 16 de los 36 países de América Latina y el Caribe. En los menores de 4 meses, la lactancia se clasificó como exclusivamente materna, parcialmente materna o no materna, y en los lactantes de 4 a 12 meses como parcialmente materna o no materna. Se utilizaron métodos de riesgos atribuibles para calcular la fracción de la mortalidad por enfermedades diarreicas e infecciones respiratorias agudas que podrían ser evitadas por la lactancia exclusivamente materna de los niños menores de 4 meses y por la lactancia parcialmente materna de los niños de 4 a 12 meses. La población de lactantes cubierta por este estudio fue del $87,2 \%$ en el Caribe, del $95,6 \%$ en Centroamérica y del $82,1 \%$ en Sudamérica.

Los resultados obtenidos revelaron que el 55\% (intervalo de confianza del 90\%: 36 a $66 \%$ ) de las muertes de lactantes latinoamericanos por enfermedades diarreicas (61\%) e infecciones respiratorias agudas $(51 \%)$ serían evitables con la lactancia exclusivamente materna de los menores de 4 meses y con la lactancia parcialmente materna de los lactantes de 4 a 12 meses. La lactancia exclusivamente materna de los menores de 4 meses evitaría el $66 \%$ (intervalo de confianza del $90 \%$ : 48 a $76 \%$ ) de estas muertes $(78 \%$ por enfermedades diarreicas y $57 \%$ por infecciones respiratorias agudas), mientras que la lactancia parcialmente materna de los lactantes de 4 a 12 meses evitaría el 32\% (intervalo de confianza del 90\%: 10 a $51 \%$ ) de las mismas (33\% por enfermedades diarreicas y $31 \%$ por infecciones respiratorias agudas). Por otro lado, el 13,9\% (intervalo de confianza del 90\%: 8,8 a $18,5 \%$ ) de las muertes por cualquier causa también serían evitadas con estas medidas, lo cual significa aproximadamente 52000 (intervalo de confianza del 90\%: 33098 a 69 320) muertes evitables en toda América Latina y el Caribe.

Para las enfermedades diarreicas, la mortalidad evitable en lactantes de menos de 4 meses osciló entre 0,84 en Cuba y 0,57 en Perú, y para las infecciones respiratorias agudas entre 0,66 en Trinidad y Tabago y 0,43 en Perú. En ambos casos, cerca de la mitad de las muertes evitables ocurrieron en Brasil y México, los dos países más poblados de la Región.

De acuerdo con estos resultados, los autores concluyen que la lactancia exclusivamente materna 
de los niños de menos de 4 meses y la lactancia parcialmente materna de los de 4 a 12 meses podría reducir sustancialmente la mortalidad infantil en América Latina y el Caribe. Como la mayor reducción potencial de la mortalidad infantil correspondió a los menores de 4 meses, las intervenciones destinadas a promover la lactancia materna deberían centrarse sobre todo en este grupo de edad. (Betrán AP, De Onís M, Lauer JA, Villar J. Ecological study of effect of breast feeding on infant mortality in Latin America. BMJ 2001;323:303-306.)

\section{Impacto del sobrepeso en el riesgo de enfermedades crónicas frecuentes}

En los Estados Unidos de América, donde el $32 \%$ de los adultos tienen sobrepeso y un $22,5 \%$ adicional son obesos, la obesidad constituye un grave problema de salud pública. El sobrepeso y la obesidad constituyen factores de riesgo de enfermedades cardiovasculares, de algunos cánceres, de diabetes y de mortalidad, además de exacerbar muchas otras enfermedades crónicas, como la hipertensión, la artrosis, la litiasis biliar, las dislipemias y los problemas musculoesqueléticos. Lamentablemente, la comparación de los diferentes estudios epidemiológicos es difícil debido a la ausencia de consenso sobre las categorías de peso, complicada por el hecho de que tanto las directrices dietéticas de los Estados Unidos como de la Organización Mundial de la Salud han cambiado la definición del sobrepeso, que ahora consiste en un índice de masa corporal (IMC) $\geq 25$, en vez de 27,3 para las mujeres y 27,8 para los hombres, como era antes.

Con el fin de investigar el impacto del sobrepeso en diferentes enfermedades crónicas, los autores de este estudio analizaron los riesgos de hipercolesterolemia, hipertensión, litiasis biliar, diabetes de tipo 2, cardiopatías, accidentes cerebrovasculares y cáncer de colon al cabo de 10 años (1986 a 1996) en los participantes de dos grandes estudios de cohortes prospectivos realizados en Estados Unidos: el Nurses' Health Study, en mujeres, y el Health Professionals Follow-up Study, en hombres.

El promedio de edad fue similar en ambas cohortes (52,9 en las mujeres y 54,5 en los hombres) y en ambas predominaron los individuos de raza blanca. Sin embargo, la prevalencia de la obesidad (IMC $\geq 30,0$ en 1986) y del consumo actual de tabaco fue más elevada en las mujeres que en los hombres $(14,8$ frente a $8,2 \%$, y 21,1 frente a $9,9 \%$, respectivamente).

El riesgo de presentar diabetes, litiasis biliar, hipertensión, cardiopatías y accidentes cerebrovasculares aumentó con la gravedad del sobrepeso en ambas cohortes. Tanto los hombres como las muje- res con un IMC $\geq 35,0$ tuvieron un riesgo cerca de 20 veces mayor de sufrir diabetes que los individuos de su mismo sexo con IMC de 18,5 a 24,9. Además, los individuos con sobrepeso, pero no obesos (IMC de 25 a 29,9) también tuvieron un riesgo 3 veces mayor de presentar diabetes a lo largo de los 10 años de estudio, en comparación con aquellos cuyo IMC era normal.

En las mujeres con sobrepeso, pero no obesas, también se detectó mayor riesgo de litiasis biliar (riesgo relativo: 1,9), hipertensión (riesgo relativo: 1,7), hipercolesterolemia (riesgo relativo: 1,1) y cardiopatías (riesgo relativo: 1,4). El riesgo de cáncer de colon y accidentes cerebrovasculares también estaba aumentado, aunque de forma no significativa. En los hombres se obtuvieron resultados similares, con la diferencia de que el aumento del riesgo de accidentes cerebrovasculares fue estadísticamente significativo (riesgo relativo $\geq 2,0$ ).

Es de señalar también que la relación dosisrespuesta entre el IMC y el riesgo de presentar enfermedades crónicas fue evidente incluso en los individuos con un IMC en los límites superiores de la normalidad $(22,0$ a 24,9$)$.

A juicio de los autores, estos resultados indican que los adultos deberían intentar mantener su IMC entre 18,5 y 21,9, con el fin de reducir el riesgo de padecer enfermedades crónicas. (Field AE, Coakley EH, Must A, Spadano J, Laird N, Dietz WH. Impact of overweight on the risk of developing common chronic diseases during a 10-year period. Arch Intern Med 2001;161:1581-1586.)

\section{La obesidad: causa importante de morbilidad}

Según una encuesta telefónica domiciliaria de una muestra de más de 9500 adultos estadounidenses, la cual fue realizada por investigadores de la organización RAND (abreviación de research and development) en Santa Mónica, California, la obesidad recibe mucha menos atención que el tabaquismo o alcoholismo como factor de riesgo para la salud. No obstante, hoy en día hay muchos más adultos obesos en Estados Unidos que fumadores o personas que abusan del alcohol.

Los investigadores recolectaron datos de adultos en distintas partes del país con respecto a talla, peso, ingresos, hábitos tabáquicos, consumo de alcohol, y afecciones crónicas. Un análisis de regresión logística de la información así obtenida reveló que el exceso de peso $(25 \leq$ IMC $\leq 30)$ y la obesidad (IMC $\geq$ 30) tienen alta prevalencia - se detectaron en 36 y $23 \%$ de los encuestados, respectivamenrte- y que la obesidad se asocia con más morbilidad, entendida en términos de la presencia de afecciones crónicas que menoscaban la calidad de vida, que el taba- 
quismo y el alcoholismo. Las personas que habían fumado toda la vida y que vivían en la pobreza mostraron una tendencia significativamente mayor $(P<0,01)$ a sufrir enfermedades crónicas, como diabetes, asma, enfermedad coronaria y artritis. En cambio, los efectos de la pobreza y del tabaquismo sobre la salud fueron menos pronunciados, una vez efectuados los debidos ajustes poblacionales, que los de la obesidad, ya que el porcentaje de personas obesas en la muestra encuestada $(23 \%)$ fue mucho mayor que el de personas pobres (14\%), el de personas que consumían bebidas alcohólicas en exceso $(6 \%)$ y el de personas que fumaban diariamente $(19 \%)$. Está confirmado que la obesidad se asocia con un aumento del costo de la atención de salud, un aumento de la mortalidad por todas las causas y un riesgo aumentado de enfermedad coronaria, osteoartritis, diabetes mellitus, hipertensión, dislipemias y ciertos tipos de cáncer.

Estos resultados resaltan la necesidad de poner en marcha programas dirigidos a eliminar la obesidad y de otorgar atención prioritaria a escala nacional a programas para el control del peso corporal, especialmente en vista de que la obesidad está aumentando en todos los estratos de la sociedad. (Sturm R, Wells KB. Does obesity contribute as much to morbidity as poverty or smoking? Public Health 2001;115:229-235.)

\section{Morbilidad y mortalidad por malaria e impacto económico de la enfermedad}

En un número suplementario de American Journal of Tropical Medicine and Hygiene, un grupo de expertos publica información que arroja nueva luz sobre la carga de malaria en el mundo y sus consecuencias económicas y sociales. Según afirma Gerald $\mathrm{T}$. Keusch, director del Fogarty International Center (FIC) y de la Multilateral Initiative on Malaria (MIM), es imprescindible cuantificar, en términos epidemiológicos y económicos, la enorme carga que impone la malaria a la familia y la sociedad. La razón de ser del MIM, según explica, radica en la gran disparidad de las iniciativas de investigación sobre la malaria, que ha llevado a los científicos de Estados Unidos, Europa y África a aunar esfuerzos para promover las actividades de investigación en el continente africano y para idear intervenciones nuevas y más eficaces para combatir la enfermedad.

Durante medio siglo ha circulado el dicho de que "cada año la malaria cobra un millón de vidas humanas". No obstante, hasta hace muy poco nadie había tratado de examinar en serio la exactitud de esta estimación, ni sus repercusiones clínicas y económicas. Según los datos publicados en el suplemento de Am J Trop Med Hyg, de 700000 a 2,7 millones de personas mueren anualmente de malaria, y más de $75 \%$ de ellas son niños africanos. Esos mismos datos indican que más de $85 \%$ de estas defunciones infantiles ocasionadas por la malaria se asocian con estados anémicos, bajo peso al nacer e hipoglicemia.

De 400 a 900 episodios agudos de fiebre se producen cada año en el África en niños menores de 5 años que viven en zonas endémicas. Se calcula que para 2020 esta cifra se duplicará si no se ponen en marcha medidas de control eficaces. El problema es especialmente serio debido a que en niños pequeños la fiebre muy alta, que a menudo se acompaña de convulsiones, puede atrasar el desarrollo cerebral y afectar a las funciones cognoscitivas más complejas, que son las responsables de la habilidad para planificar, tomar decisiones, ser consciente del comportamiento propio y tener sensibilidad social. De todas las manifestaciones de la malaria, las que menoscaban las funciones cognoscitivas y la conducta son las más sutiles e indefinidas y las que más profundamente perjudican a los niños, a la familia $\mathrm{y}$ a la sociedad en general.

El suplemento también aborda el efecto que ejerce la malaria sobre el desarrollo económico y social. Por ejemplo, el incremento del ingreso per cápita de 1965 a 1990 en países donde hay un grave problema de transmisión de la malaria fue únicamente $0,4 \%$ al año, a diferencia de $2,3 \%$ en países con un índice relativamente bajo de infecciones maláricas. (The intolerable burden of malaria: a new look at the numbers. Am J Trop Med Hyg 2001; February (suppl).) 